\title{
Post-operative intensive care: Is it really necessary?
}

RMJ Cashmore ${ }^{1}$, AJ Fowler ${ }^{1}$, RM Pearse ${ }^{1}$

1. Critical Care and Perioperative Medicine Research Group, Queen Mary University of London.

Correspondence and reprint requests to:

Professor Rupert Pearse

Adult Critical Care Unit

Royal London Hospital

Whitechapel Road

Whitechapel

London

E1 1BB

rpearse@qmul.ac.uk

Word count: 1165 [excluding figures]

Figures: 1

References: 19

Keywords: Intensive care; surgery; post-operative; perioperative medicine.

ORCID IDS

RMJC: 0000-0002-0266-4308

AJF: 0000-0002-1491-7739

RMP: 0000-0002-4373-5934 
It is estimated that more than 310 million patients receive a surgical treatment each year [1], and noted that the number of procedures performed each year is growing [2-6]. Despite a majority of cases being performed without significant complication, deaths after surgery have recently been demonstrated to account for a large portion of all deaths worldwide [7]. For a minority of patients, surgical procedures carry a significant burden of both death and disability. High-risk patients account for approximately $10-15 \%$ of the surgical population, but suffer around $80 \%$ of post-operative deaths [4][8]. These high-risk patients have only been loosely defined, being typically older with a higher burden of comorbid disease. The surgical population is ageing at a faster rate than the background population [3]. With increasing numbers of procedures on increasingly high-risk patients, there is a clear need to identify possible interventions that improve peri-operative outcomes [4][9], with many targeted at the high-risk group who stand to benefit the most.

A number of potential therapies have been proposed and investigated, including pharmacological, physiological and multimodal interventions to improve post-surgical outcomes. The challenge remains to personalise these interventions to those surgical patients most likely to benefit $[10,11]$. Some interventions need to be targeted at carefully identified patient groups, whilst others are not cost effective in low-risk populations. Enhanced Recovery After Surgery (ERAS) pathways focus on protocolizing evidence-based interventions within defined surgical populations. These can improve outcomes whilst reducing burdens on healthcare systems [12]. At present, the high-risk non-cardiac surgical patient is much harder to identify within the greater surgical population, and move to an alternative patient pathway.

Post-operative admission to the Intensive Care Unit (ICU) is itself considered a standard of care in many healthcare systems [2]. Patients with major acute, yet widely varying primary pathologies, find themselves admitted to the ICU for generally similar supportive therapies. As physicians, this leads us to regard ICU as the gold-standard of high-risk and post-operative care. That said, ICU resources are 
expensive, finite, and in ever-increasing demand [5][13]. The need to minimize unnecessary and inappropriate admission to critical care is important in all healthcare systems. It is of even greater importance in low resource settings, where critical care bed availability per captia is significantly less. Inappropriate allocation impacts not only the high-risk surgical population, but other cohorts concurrently needing critical care resources. Subsequently, the overall effects at a population level are negatively amplified.

Surgical patients continue to make up a sizeable proportion of ICU admissions [2], however, there is wide variation in admission rates to ICU after non-cardiac surgery, suggesting a lack of consensus on its optimal use [9][14][15]. Failure-To-Rescue (FTR) is of particular relevance and concern being a term used to describe those who die from early postoperative complications [15]. FTR may demonstrate current system weaknesses, with longer time periods before the identification and treatment of complications resulting in greater mortality [16]. The chronology of post-operative medical and surgical complications are of importance when considering delivery of any post-operative intervention. An intervention designed to either prevent, or promptly recognise and respond to a complication, must be provided at a time when the risk/frequency of occurrence is highest. These high-risk periods are not yet clearly defined, and ICU re-admission rates suggest further research in this area may be beneficial.

The potential missed opportunity for intervention is in line with findings that indirect post-operative admission to an ICU is associated with increased peri-operative and long-term mortality [15]. Figure 1 shows some key factors influencing current post-operative care. Secondary to global differences in capacity, culture and structure, there is variation in the patient demographics of admissions to ICU. In a cohort of mixed United States ICU's, almost $40 \%$ of all admissions were for monitoring purposes only [17], whereas in the UK, patients admitted to critical care tend to require higher levels of organ support. 
The boundaries of exactly what does and does not constitute Intensive care remains a matter of debate, and described international variations further compound any data-analysis [16][17]. This is additionally complicated by the varied nomenclature used to describe the different critical care environments. For example, locations currently termed 'High Dependancy Units (HDUs)', 'Step-down Facilities' and/or 'Post-operative Anaesthesia Care Units (PACUs)' often offer similar capabilities. Some of the key aspects of more classical 'Intensive Therapy Units' are higher staff-to-patient ratios, integrated multi-disciplinary team (MDT) approaches, provision of advanced physiological support techniques, frequency of experienced clinician input, and enhanced monitoring crucially combined with rapid-response interventions.

Most post-operative patients do not require the full complement of ICU interventions available, and those interventions that are required could reasonably be delivered on a well-resourced ward with clear cost-saving implications. Indeed, key to most surgical patients' recovery, are relatively simple interventions such as analgesia, early mobilisation, and early identification combined with treatment of complications [15]. Several ongoing multi-centre clinical trials are exploring the benefits of typical peri-operative ICU interventions which have been modified to be delivered outside of the ICU. Notably, by simply having increased numbers of nursing and medical staff available per patient in an ICU environment may have important effects on outcomes. In the UK, organisations with a low 30 day mortality rate had significantly greater provision per bed of doctors and nursing staff [18]. In this regard, ICU is an expensive fix to more widespread system issues. The exact mechanism, however, by which greater use of critical care may improve outcomes after high risk surgery remains undefined [19].

Recently published results from the EPOCH trial [11] did not identify any survival benefit from a national quality improvement programme for patients undergoing emergency abdominal surgery. It 
highlighted the complexity of modern hospital care pathways, and the difficulties in both implementing broad changes and measuring outcome. In many ways, the post-operative ICU concept is similar to this. In line with recommendations from $\mathrm{EPOCH}$, building an evidence base for individual interventions will likely be key to improving outcomes. Once established, these proven individual interventions can sequentially be placed into the overall process of care pathway in various guises as appropriate.

\section{Conclusion}

Intensive care is not an intervention that can be easily tested in a randomised trial, relying instead on observational data and the inherent difficulties this presents. Research into early identification of the high-risk population for whom interventions could be targeted for a maximal dose-response will aid understanding of who could benefit the most from post-operative ICU admission and facilitate improved resource utilization. The patient-societal balance in high, middle, and low income countries may have further influence on resource utilization. Investigation into specific peri-operative interventions continues to provide the essential evidence base to help clarify the role of the individual components of post-operative ICU care. As clear evidence-based interventions are established, the location of where these interventions are delivered in a hospital system can then be addressed. In the interim, ICU continues to function as an attractive solution. For the future, we need to understand the question we are asking of post-operative ICU before we try to assess and rationalise the solutions it provides.

\section{Conflicts of interest}

RP holds research grants, has given lectures, and/or performed consultancy work for Nestle Health Sciences, BBraun, Medtronic, Glaxo SmithKline, Intersurgical, and Edwards Lifesciences. All other authors declare no conflicts of interest. 


\section{Funding}

RP is supported by an NIHR Research Professorship. AJF is supported by an NIHR Doctoral Research Fellowship (DRF-2018-11-ST2-062). 


\section{Figure legends}

Figure one. Flow diagram indicating post-operative care factors 


\section{References}

1. Weiser TG, Haynes AB, Molina G, et al (2015) Estimate of the global volume of surgery in 2012: an assessment supporting improved health outcomes. Lancet 385:S11. https://doi.org/10.1016/S0140$6736(15) 60806-6$

2. Gillies MA, Sander M, Shaw A, et al (2017) Current research priorities in perioperative intensive care medicine. Intensive Care Med. 43:1173-1186

3. Fowler AJ, Abbott TEF, Prowle J, Pearse RM (2019) Age of patients undergoing surgery. BJS bjs.11148. https://doi.org/10.1002/bjs.11148

4. Pearse RM, Holt PJE, Grocott MPW (2011) Managing perioperative risk in patients undergoing elective non-cardiac surgery. BMJ 343

5. Ghaffar S, Pearse RM, Gillies MA (2017) ICU admission after surgery. Curr Opin Crit Care 23:424-429. https://doi.org/10.1097/mcc.0000000000000448

6. Edwards MR, Forbes G, MacDonald N, et al (2019) Optimisation of Perioperative Cardiovascular Management to Improve Surgical Outcome II (OPTIMISE II) trial: study protocol for a multicentre international trial of cardiac output-guided fluid therapy with low-dose inotrope infusion compared with usual care in patients undergoing major elective gastrointestinal surgery. BMJ Open 9:e023455. https://doi.org/10.1136/bmjopen-2018-023455

7. Nepogodiev D, Martin J, Biccard B, et al (2019) Global burden of postoperative death. Lancet (London, England) 393:401

8. Pearse RM, Moreno RP, Bauer P, et al (2012) Mortality after surgery in Europe: A 7 day cohort study. Lancet 380:1059-1065. https://doi.org/10.1016/S0140-6736(12)61148-9

9. Kahan BC, Koulenti D, Arvaniti K, et al (2017) Critical care admission following elective surgery was not associated with survival benefit: prospective analysis of data from 27 countries. Intensive Care Med 43:971-979. https://doi.org/10.1007/s00134-016-4633-8

10. Pearse R, Dawson D, Fawcett J, et al (2005) Early goal-directed therapy after major surgery reduces complications and duration of hospital stay. A randomised, controlled trial [ISRCTN38797445]. Crit Care 9:R687-93. https://doi.org/10.1186/cc3887

11. Peden CJ, Stephens T, Martin G, et al (2019) E ff ectiveness of a national quality improvement programme to improve survival after emergency abdominal surgery ( $E P O C H$ ): a stepped-wedge 
cluster-randomised trial. Lancet (London, England) 393:2213-2221

12. Ljungqvist O, Scott M, Fearon KC (2017) Enhanced recovery after surgery a review. JAMA Surg 152:292-298. https://doi.org/10.1001/jamasurg.2016.4952

13. Gillies MA, Pearse RM (2016) Intensive Care after High-risk Surgery. Anesthesiology 124:761-762. https://doi.org/10.1097/aln.0000000000001025

14. Jerath A, Laupacis A, Austin PC, et al (2018) Intensive care utilization following major noncardiac surgical procedures in Ontario, Canada: a population-based study. Intensive Care Med 44:1427-1435. https://doi.org/10.1007/s00134-018-5330-6

15. Gillies MA, Harrison EM, Pearse RM, et al (2017) Intensive care utilization and outcomes after high-risk surgery in Scotland: A population-based cohort study. Br J Anaesth 118:123-131. https://doi.org/10.1093/bja/aew396

16. Vincent J-L, Singer M (2010) Series Critical Care 3 Critical care: advances and future perspectives. Lancet 376:1354-1361. https://doi.org/10.1016/S0140

17. Murthy S, Wunsch H (2012) Clinical review: International comparisons in critical care-lessons learned. Crit Care 16:1-7

18. Ozdemir BA, Sinha S, Karthikesalingam A, et al (2016) Mortality of emergency general surgical patients and associations with hospital structures and processes. Br J Anaesth 116:54-62. https://doi.org/10.1093/bja/aev372

19. Gillies MA, Power GS, Harrison DA, et al (2015) Regional variation in critical care provision and outcome after high-risk surgery. Intensive Care Med 41:1809-1816. https://doi.org/10.1007/s00134015-3980-1 